\title{
PROPAGAZIONE DELLA MAREA NELLA LAGUNA DI VENEZIA
}

\author{
Silvio Polli
}

1. Premesse. - La laguna di Venezia si estende lungo una fascia costiera leggermente arcuata per una lunghezza di $50 \mathrm{~km}$ e con una larghezza media di circa $12 \mathrm{~km}$. Verso il mare è limitata da un cordone litoraneo nel quale si aprono tre bocche o porti-canale. Mediante questi la laguna ccmunica con il mare. Esternamente al cordone litoraneo, nel mare aperto, l'onda di marea si propaga da NNE a SSW senza notevoli ritardi, in modo che essa si presenta alle tre bocche quasi contemporaneamente. All'imbocco del porto canale di Chioggia vi arriva 4 minuti dopo il transito davanti a quello del Lido.

Attraverso i tre porti-canale la marea si espande nella laguna diramandosi lungo i numerosi canali. Questi sono in parte sempre sommersi e in parte coperti dall'acqua solo durante le alte maree. Le sezioni dei canali decrescono allontanandosi dalle bocche. Il canaleporto di Lido è largo $800 \mathrm{~m}$ ed ha una profondità media di $8 \mathrm{~m}$, quello di Malamocco ha una larghezza di $420 \mathrm{~m}$ e fondali medi di $12 \mathrm{~m}$ e quello di Chioggia è largo $500 \mathrm{~m}$ e profondo in media 7 . tlle loro estremiti, ai margini della laguna, le sezioni dei canali arrivano a qualche m" e anche meno. La lunghezza di alcuni di questi canali supera i $20 \mathrm{~km}$. Essi determinano allora forti ritardi di fase e notevoli riduzioni di ampiezze.

La propagazione della marea avviene essenzialmente lungo i canali, i vasti e hassi fondali della laguna viva e morta smorzano rapidamente il moto di propagazione. Durante il flusso l'acqua dilaga dai canali nelle zone lagunari, clevandone il livello, in epoca di sizigie, dai 40 agli $80 \mathrm{~cm}$. Estese zone che rimangono scoperte durante le hasse maree vengono allora sommerse. La forma del hacino varia noterolmente e periodicamente.

Durante il riflusso l'acqua affluita si ritira nei canali, e, lungo questi, al mare. La propagazione della marea nella laguna risulta dunque un fenomeno complesso. I fattori principali che lo determinano sono: la lunghezza, la sezione, la tortuosità, la natura del fondo e 
l'intreccio dei canali. Il fatto che molto spesso il canale, pur avendo un letto proprio, manca di sponde solide (in quanto scorre nelle acque lagunari) complica maggiormente la ricerca.

Elementi fondamentali per la conoscenza e per ogni studio delle maree, sia di una regione marina che lagunare, sono le costanti armoniche delle maree determinate per un numero sufficientemente grande di siti. Perciò, prima di passare a sviluppi dimostrativi, relativi al comportamento delle maree, occorre eseguire il calcolo delle costanti armoniche. Queste sono state da noi calcolate per 12 località della laguna. Esse, assieme a quelle di altre 6 stazioni precedentemente determinate da altri autori, costituiscono un complesso di analisi corrispondenti a 18 località lagunari. Questi dati, se anche possono essere pochi per uno studio esauriente e dettagliato del fenomeno, danno già una visione generale delle condizioni mareografiche lagunari. Per i siti esaminati le maree risultano invece precisate in ognı particolare.

In questo lavoro si presentano: tutte le costanti armoniche e non armoniche sinora determinate per la laguna; le linee di uguale media altezza delle alte maree sizigiali tracciate di 5 in $5 \mathrm{~cm}$; le linee di uguale stabilimento del porto di 30 in 30 minuti; le linee di uguale situazione locale della componente semidiurna principale $M$. di 10 in 10 gradi.

Il calcolo delle costanti armoniche per 12 localita ha richiesto notevole impiego di lavoro e di tempo. Esso è stato eseguito presso l'Istituto Talassografico di Trieste e ultimamente presso la Sezione Mareografica del Centro Studi Talassografici del C.N.R. Quest'ultimo ha contriluuito notevolmente alla ricerca col mettere a disposizione sia un calcolatore che due macchine calcolatrici. Si ringrazia il Direttore dell'Istituto e del Centro Studi, prof. F. Vercelli, che agrevolò in tutti i modi queste ricerche.

2. - Le Stazioni mareografiche. - L'Ufficio Idrografico del Magistrato alle Acque di Venezia cura la sistemazizone e la manutenzione di tutti i mareografi della laguna. Per le principali stazioni esso elabora i dati delle alte e delle basse maree e li pubblica nel "Bolletiino Mensile " e negli "Annali Idrologici ". I mareogrammi sono poi conservati nell'archivio dell'Ufficio. Per i nostri calcoli sono stati messi a disposizione i diagrammi originali e di ciò siamo grati al Direttore ingr. L. Vollo. Lo slesso Ufficio ha provveduto al collegamento delle 
piastrine dei marcografi con i capisaldi della rete altimetrica dello Stato.

Nella zona lagunare hanno funzionato successivamente oltre una cinquantina di mareografi. Alcuni per necessita di carattere generale, altri per ricerche particolari occorrenti al Magistrato alle Acque. Dei principali di essi, e precisamente per 18 stazioni, sono state determinate le costanti armoniche e non armoniche delle maree. $\grave{E}$ in corso il calcolo per un altro sruppo di stazioni interne.

Nella tabella I sono elencate le stazioni considerate in questo lavoro, ordinate secondo la latitudine decrescente. Le loro coordinate geografiche sono state determinate dalle carte alle scale 1:25000 e 1:100000 dell'Istituto Geografico Militare, secondo le indicazioni sulle posizioni delle stazioni avute dall'Ufficio Idrografico. Le longitudini sono riferite al meridiano di Greenwich, per averle rispetto a quello di M. Mario (Roma) hasta togliere da esse l'angolo di 12' $27^{\prime} 08^{\prime \prime}$, valore da noi usato nella trasformazione. Nella colonna "N. mesi” è indicato il periodo di registrazione analizzato nel calcolo delle costanti armoniche. La colonna successiva dà il corrispondente anno. I due casi degli anni 1923-24 si riferiscono all'intervallo costituito dallultimo trimestre del 1923 e dal primo trimestre 1924. Quando di una determinazione di costanti armoniche esista una pubblicazione, questa si troveri indicata nella hibliografia sotto il nome dellautore.

Nella tabella II è indicato il tipo di mareografo in funzione all'epoca della determinazione delle costanti, il suo rapporto di riduzione, lo scorrimento del foglio in $\mathrm{mm}$ per ora ed alcuni cenni sulla posizione dello strumento.

Nella fig. 1 le 18 stazioni marcografiche sono indicate con grli stessi numeri progeressivi della tabella I. Sono state marcate le arterie principali lungo le quali la marea si propaga dalle bocche alle singrole stazioni.

Il mareografo di Porto Piave Vechia è situato fuori della laguna, sulla sponda destra della Piave Vecchia, poco a valle del ponte. circa a $1,5 \mathrm{~km}$ dalla foce. Non è in comunicazione con la laguna, risente pertanto solamente della marea che si propaga attraverso la foce. La vicina stazione mareografica di Cavallino è situata invece nella lag̣una. L'onda di marea vi perviene attraverso il Canale-Porto di Lido, percorrendo i canali Tre Porti e di Pordelio, dopo $16 \mathrm{~km}$ di percorso lagunare. Non è in libera comunicazione con la Piave Vecchia, ma un sistema di due porte automatiche permette che una parte dell'acqua 
lagunare si scarichi nel fiume. Ciò però non altera in modo apprezzabile il regime marcourafico delle due stazioni.

I porti-canali di Lido, Malamocco e di Clioggia hanno ciascuno

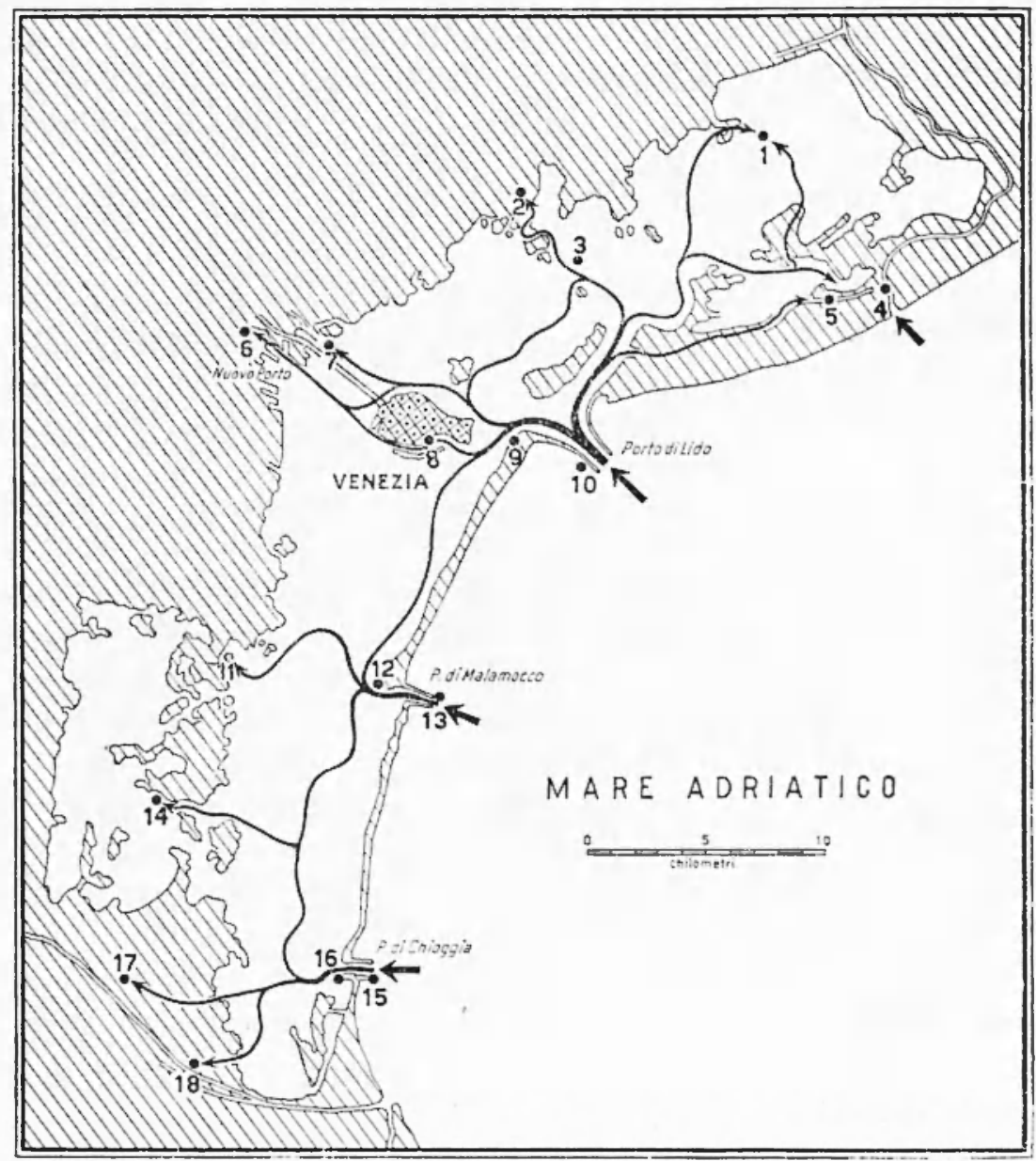

Fig. 1 - Arterie principali di propagazione della marea dalle bocche alle stazioni mareografiche

due mareografi, uno sulla diga della bocca esterna, nel mare libero, e l'altro alla bocca interna, nella laguna. Questa disposizione è necessaria perché, come si vedrà più avanti, la marea, nell'attraversamento dei porti-canale, subisce una riduzione di ampiezza e un notevole ritardo nella propagazione.

I percorsi, per le stazioni più interne, si aggirano sui $15-20 \mathrm{~km}$. 


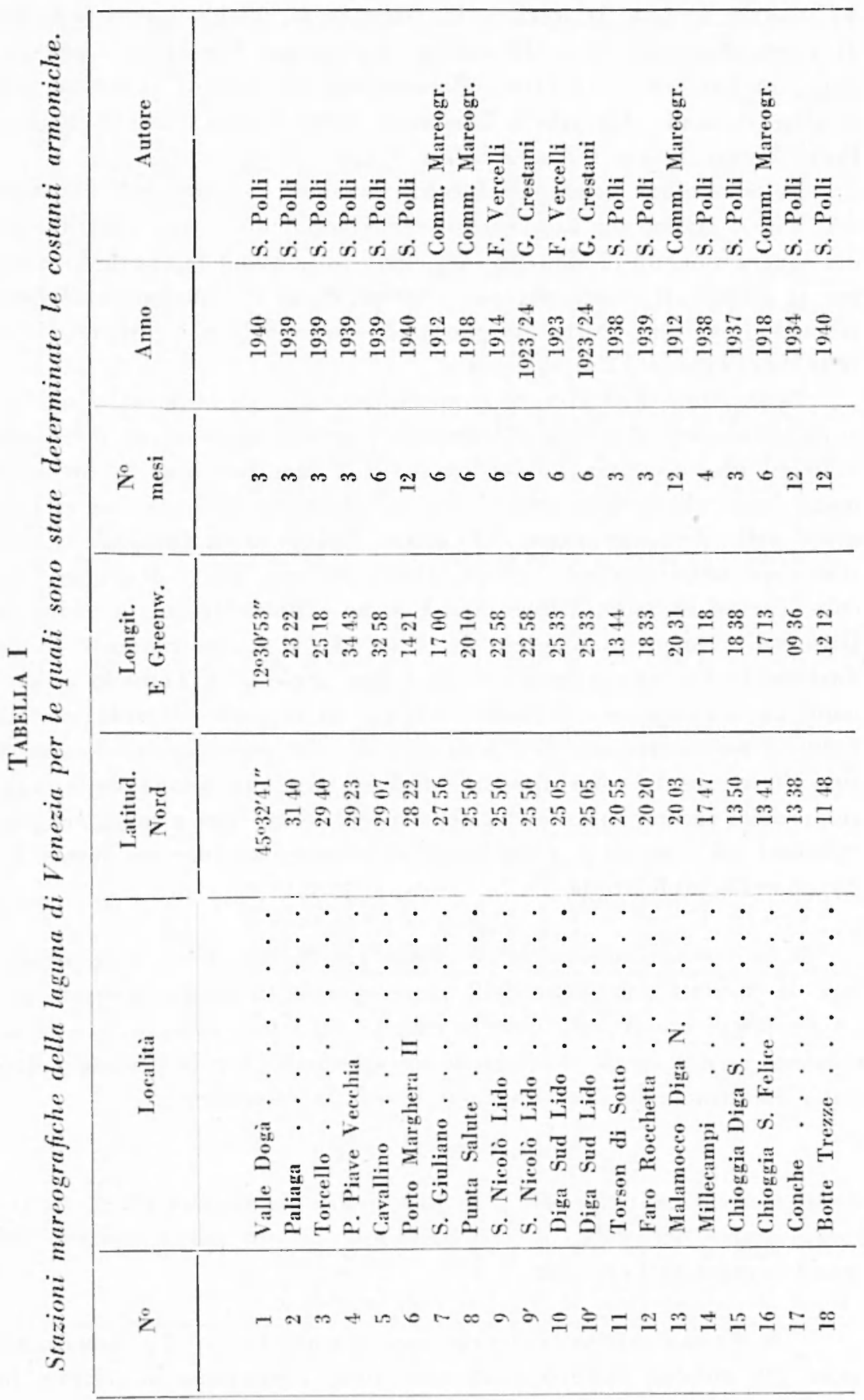


Vi sono $20 \mathrm{~km}$ per la stazione di Valle Dogà, $18 \mathrm{~km}$ per le stazioni di Porto Marghera II e Millecampi, $16 \mathrm{~km}$ per Cavallino e per Paliaga, $15 \mathrm{~km}$ per S. Giuliano. Percorsi un po' minori si hanno per le altre stazioni: Torcello e Torson di Sotto $12 \mathrm{~km}$, Conche $11 \mathrm{~km}$, Botte Trezze $10 \mathrm{~km}$ e Punta Salute $9 \mathrm{~km}$.

Nelle stazioni principali furono sistemati i mareografi del tipo "M 450 ». Hanno un rapporto di riduzione 1:5 ed uno scorrimento del diagramma di $15 \mathrm{~mm}$ per ora. Essi consentono buone letture sia per le altezze di marea che per i tempi. Sono in fumzione nelle stazioni di Cavallino, Porto Marghera II, Punta Salute, S. Nicoló Lido, Diga Sud Lido e Faro Rocchetta.

Nelle stazioni di minore importanza sono stati sistemati i mareografi settimanali del tipo "Richard", con un rapporto di riduzione 1:10 ed uno scorrimento del foglio di $2 \mathrm{~mm}$ per ora. Si prestano meno bene alle letture orarie per la minore precisione che si può avere nella determinazione del tempo. Essi sono in funzione o funzionavano nelle stazioni di Valle Dogà, Paliaga, Porto di Piave Vecchia, Torson di Sotto, Millecampi, Conche e Botte Trezze. A Chiogria Diga Sud vi è un mareografo del tipo " $G 44$ », con rapporto di riduzione 1:10 e uno scorrimento di $3 \mathrm{~mm}$ per ora. A Torcello è sistemato un mareografo "Richard" avente un rapporto di riduzione di 1:20 ed un movimento di $2 \mathrm{~mm}$ per ora. Le registrazioni di questo tipo di strumento sono appena sufficienti per una buona determinazione delle costanti armoniche delle maree. Altri dati e magriori precisazioni sui mareografi considerati si potranno trovare nei lavori indicati nella bibliografia.

3. Le costanti urmoniche. - Nelle tabelle III, IV e V si presentano le costanti armoniche delle maree per le 18 localita considerate. Le semiampiezze $H$ sono date in $\mathrm{cm}$; le situazioni adatate $g$ e le situazioni vere o locali $*$ in gradi sessagesimali. Per il passaggio dall'una all'altra delle due situazioni si usò la relazione:

$$
\varkappa=g+\left(p \lambda^{0}-n^{\circ} \cdot \Lambda^{11}\right),
$$

dove: $p=$ suffisso dell'onda, $\lambda^{\prime \prime}=$ longitudine la Greenwich, $\mathbf{n}^{\prime \prime}=$ velocita oraria dell'onda, $\Lambda^{\mathrm{h}}=$ longitudine in ore del meridiano sul quale è regolato l'orologio.

4. Esame critico delle costanti armoniche. - Le determinazioni più antiche delle costanti armoniche riguardano le stazioni di 


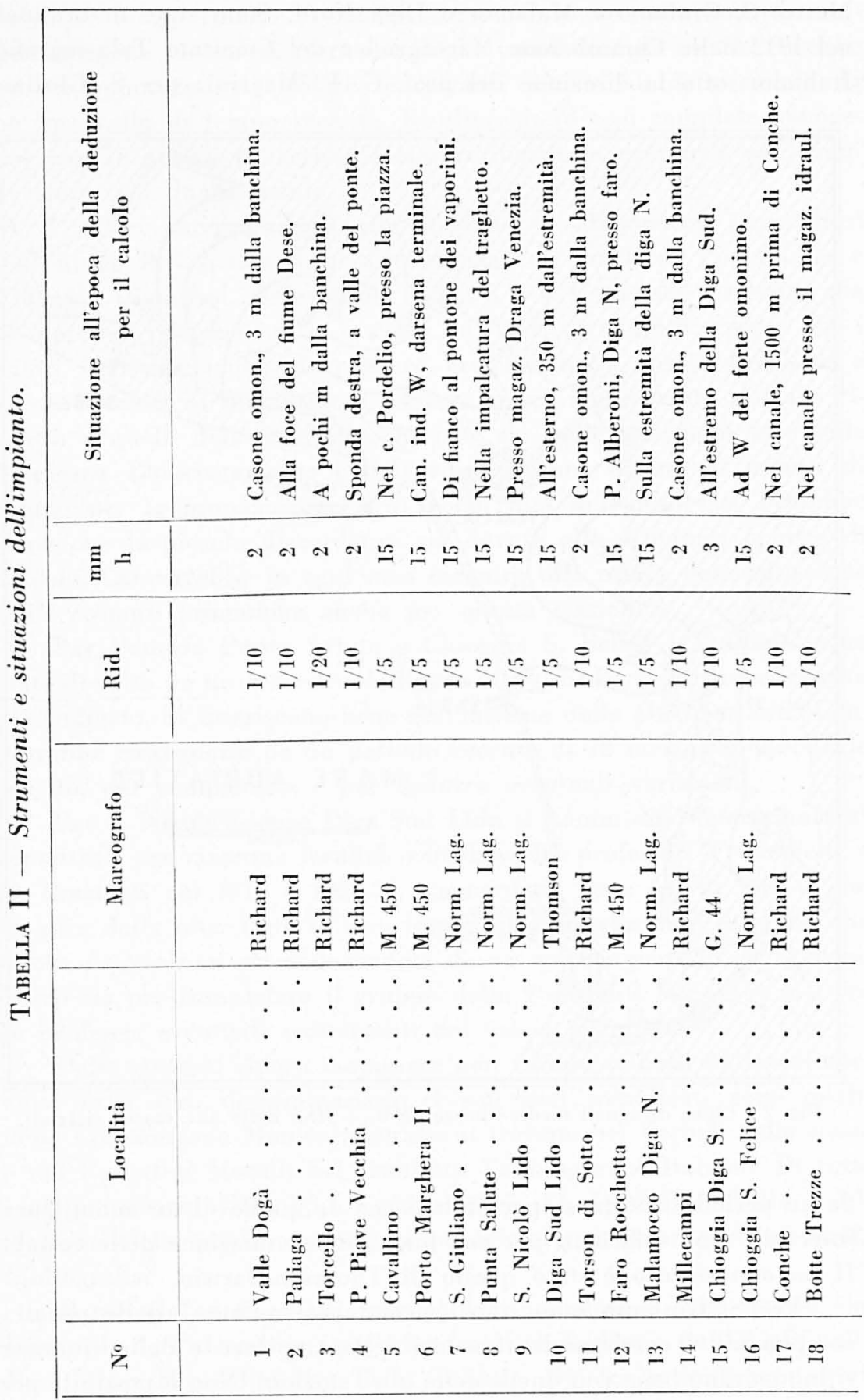


Mestre S. Giuliano e Malamocco Diga Nord. Sono state determinate nel 1912 dalla Commissione Mareografica del Comitato Talassografico Italiano, sotto la direzione del prof. G. P. Magrini; per S. Giuliano

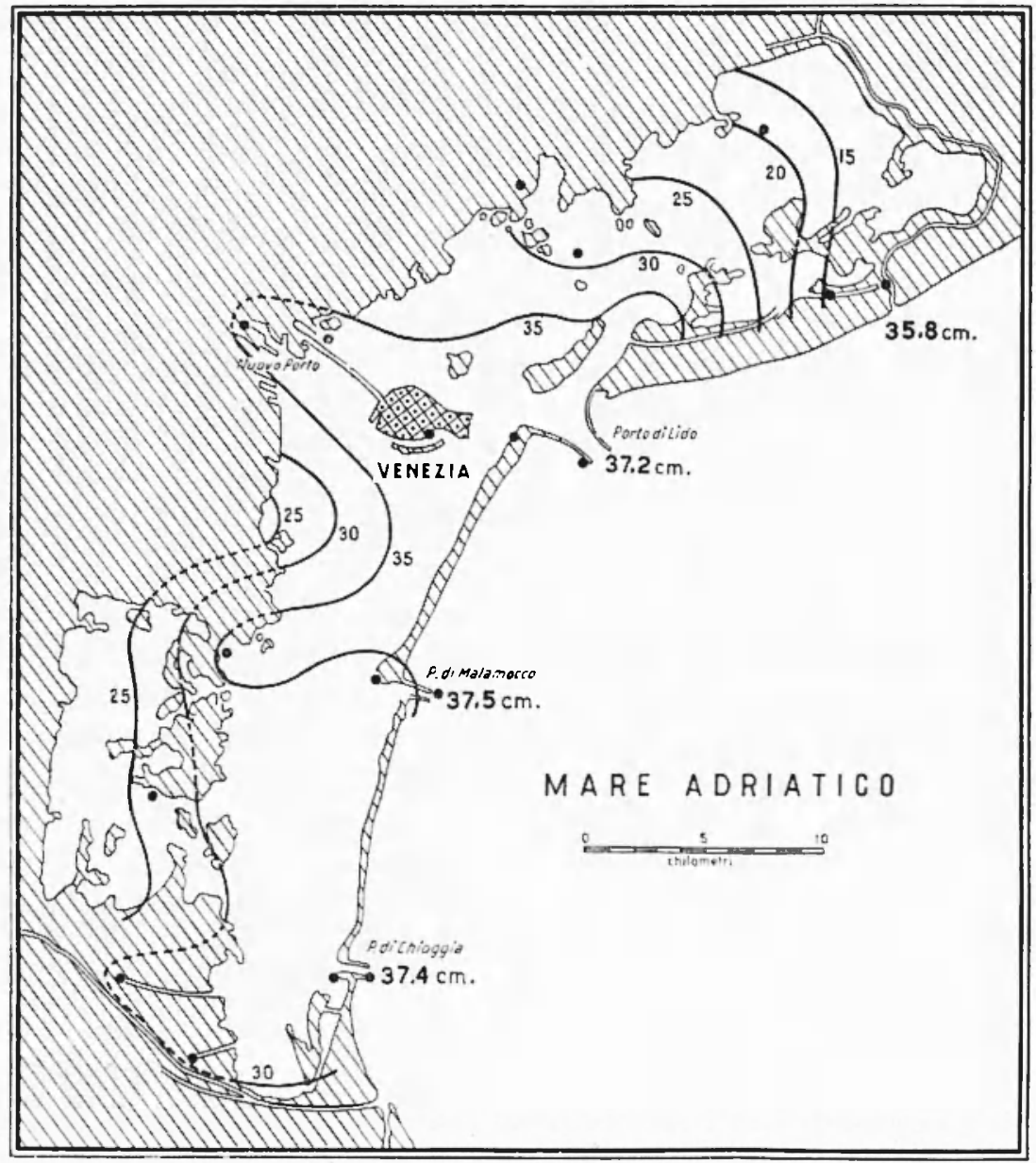

Fig. 2 - Linee di uguali medie altezze $\left(H U,+H S_{3}\right)$ delle alte maree sizigiali.

da un periodo di 5 mesi, per Malamocco da quello di un anno. Questi intervalli sono sufficienti per una buona determinazione delle costanti. 11 metodo seguito è stato quello di Thomson-Darwin.

Per S. Giuliano sono state calcolate solamente $i$ delle usuali 9 componenti. I corrispondenti valori delle ampiezze e delle situazioni si inquadrano bene con quelli delle altre stazioni. Non è possibile peri 
precisare maggiormente il confronto perché manca una stazione sufficientemente vicina ed avente analoghe condizioni di marea. Sarebbe quindi opportuno ricalcolare le costanti armoniche deducendole da un intervallo di tempo recente. Risulterebhero cosi complete, omogenee con le attuali e sarebhe facile verificare le eventuali variazioni avvenute nel quarantennio.

I valori corrispondenti alla stazione di Malamocco Diga Nord presentano invece una lieve discordanza con quelli di Porto Lido e Chioggia Diga Sud. Osservando le fig. 3 e 4 e tenendo presente che la marea si propaga lungo la costa da NNE verso SSW, risulta che i valori delle siluazioni dovrebhero crescere nella stessa direzione e verso. I valori di Malamocco figurano invece leggermente più alti rispetto a quelli delle due altre bocche, sia nclle situazioni che nelle ampiezze. Lo scostamento è dell'ordine di una decina di minuti di tempo per la propagazione e di pochi nm nell'ampiezza. Potrebbe darsi che la piccola discordanza sia dovuta alle differenti epoche di calcolo. Converreblue in ogni caso eseguire una nuova determinazione delle costanti armoniche anche per questa stazione.

Per Venezia Punta Salute e Chioggia S. Felice le costanti sono state dedotte da un semestre dell'anno 1918, mediante il procedimento già indicato. Si inseriscono bene nell'insieme delle altre costanti. Converrebbe ricalcolarle da un periodo recente di 12 mesi, per precisarle meglio, per completarle e per dedurre eventuali variazioni.

Per S. Nicolò Lido e Diga Sud Lido si hanno due determinazioni semestrali per ciascuna localiti, calcolate dai professori F. Vercelli e G. Crestanti nel 1914 e 1923-24. Concordano bene fra di loro e con le altre della rete. Data la loro importanza converrebbe eseguire una nuova determinazione deducendola da un recente periodo di 12 mesi. F ciò sia per completare il gruppo delle 9 costanti e sia per mettere in evidenza eventuali scostamenti dei valori primitivi.

Delle costanti sinora esaminate non furono pubblicati lavori specifici sulla loro determinazione. I soli dati pubblicati sono quelli della Commissione Mareografica che si trovano nei Verhali della stessa e nei Bollettini Mensili del Comitato Talassografico Italiano. Di tutte le altre stazioni (nn. 1-6, 1], 12, 14, 15, 17, 18 della tabella l) gli elementi delle analisi sono stati pubblicati nei lavori indicati nella bibliografia.

Il metodo adottato per queste determinazioni è stato quello elahorato da A. T. Doodson per l'Ammiragliato Inglese e pubblicato nella Parte III "The Admiralty Tides Tables». Dei tre procedimenti indi- 
cati si seguì quello che si applica ad una successione continua di valori orari estesa a 696 ore ( 29 giorni). Il calcolo separato per ciascun periodo di 29 giorni è conveniente per diversi motivi. Oltre che per

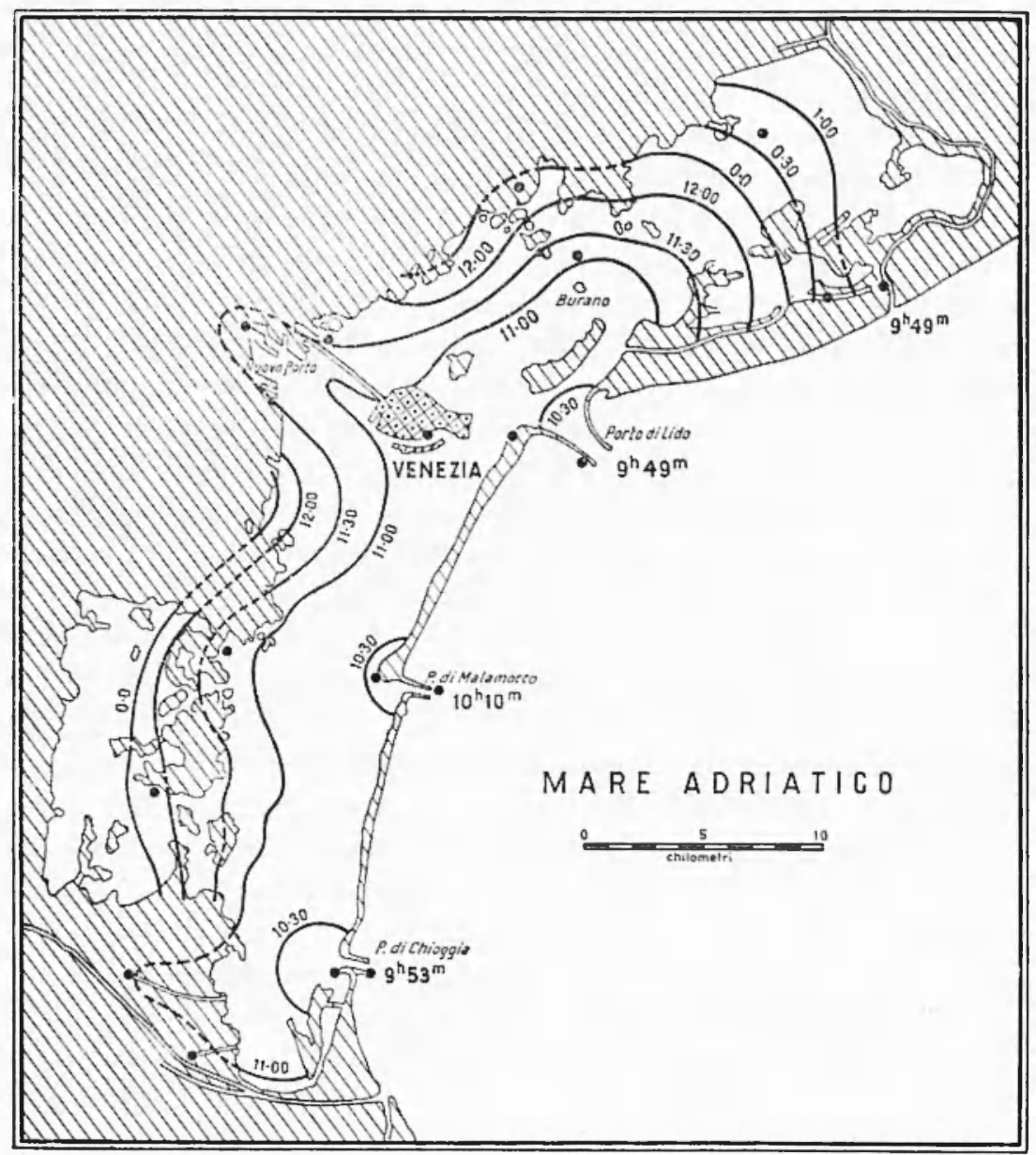

Fig. 3 - Linee di uguale stabilimento volgare del porto $\left(H H^{*} F \& C\right)$

la praticità, sveltezza e continuità di controllo dei calcoli esso è molio utile perché permette di verificare immediatamente la regolariti e la omogeneità dei risultati della serie analizzata rispetto a quelìi degli altri mesi. Infatti, scostamenti forti (per le componenti principali) fra i valori delle stesse costanti denotano errori di lettura o di calcolo oppure irregolaritì nel funzionamento del mareografo.

Quali costanti armoniche del sito esaminato si considerano le 


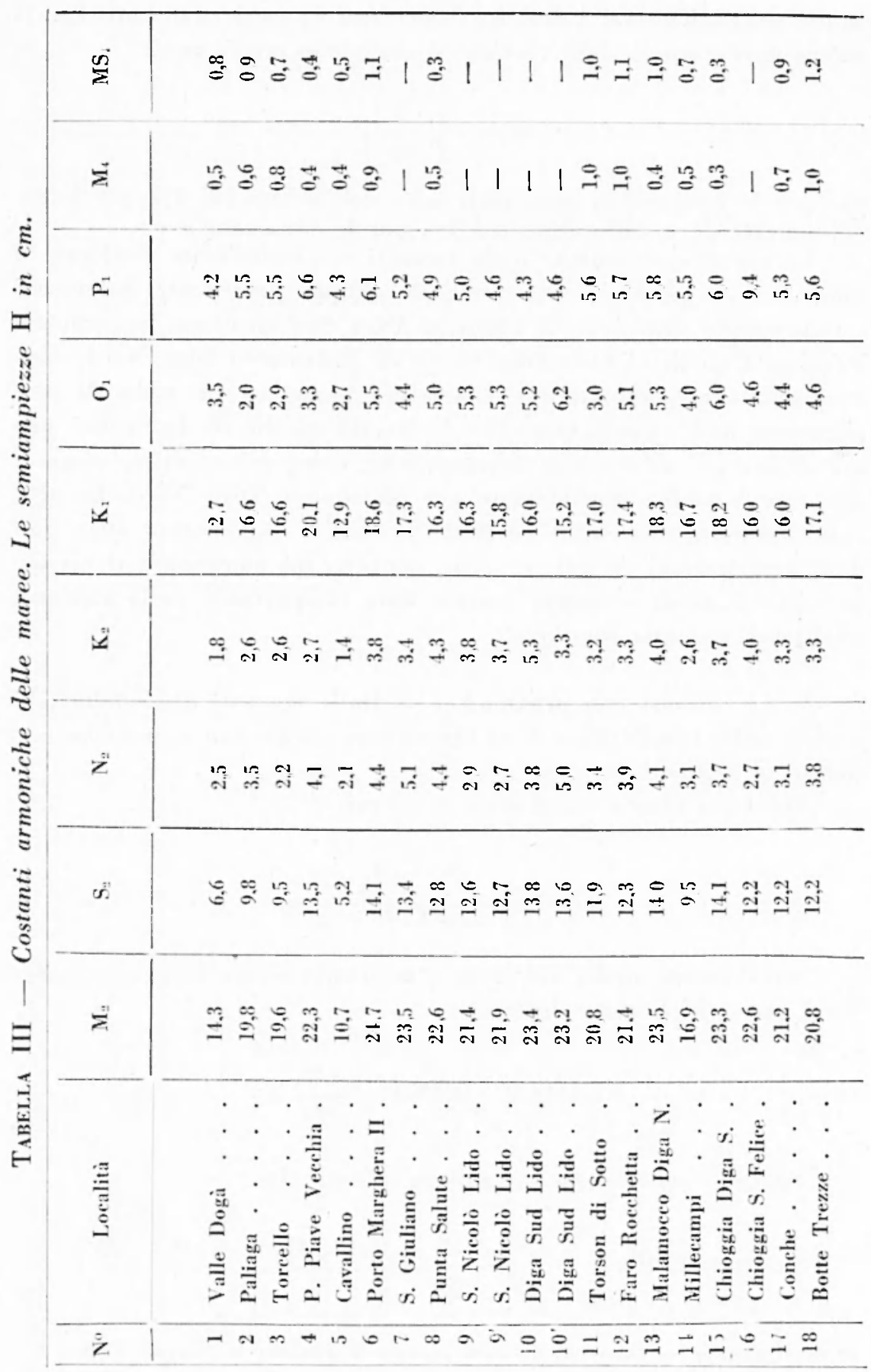


medie arimetiche dei valori corrispondenti ai mesi analizzati. Per il valore modio anmuo delle costanti si ottengrono errori medi

$$
m= \pm \sqrt{\frac{2}{n-1}}
$$

che per le componenti principali sono dell'ordine del $3 \%$ per le semiampiezze $H$, c dell'ordine dell'1\% per le situazioni $g$ e $\%$.

Le costanti armoniche delle stazioni ora considerate risultano in buon accordo fra loro e con quelle del gruppo precedente. Solamente i valori delle situazioni di Chioggia Diga Sud risultano leggermente inferiori a quelli di Lido Diga Sud e di Malamocco Diga Nord; contrariamente a quanto si dovoble avere basandosi sul verso di propagazione della marea. Ora. date le diverse epoche di deduzione, prima di cercare altre cause, hisognerebbe, come grià si disse, eseguire una nuova analisi specialmente per Malamocco Diga Nord. In ogni caso, avendosi ricavate le costanti armoniche di Chioggia Diga Sud dalle registrazioni di soli tre mesi, converreble continuare il calcolo per altri 3 mesi; o meglio ancora, data l'importanza della stazione, analizzare un anno completo.

5. Le costanti non armoniche. - Dalle costanti armoniche presentate nelle tabelle III e V si calcolarono quelle non armoniche mediante le seguenti formule.

Età della marea semidiurna in griorni:

$$
\eta=\frac{x_{1}-x_{n} M_{n}}{24^{0}, 4}
$$

Stabilimento medio del porto o intervallo modio dolle alte maree (ingl. mean high water interval):

$$
\text { IMAM }(\text { MHWI })=\frac{\varkappa \psi_{2}}{29}
$$

Tenendo conto della sovramarea $M_{4}$ di $M_{2}$ :

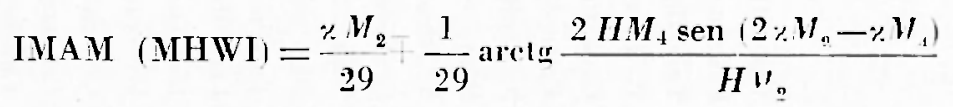

dove il segno - vale per le alte maree e quello + per le basse. 


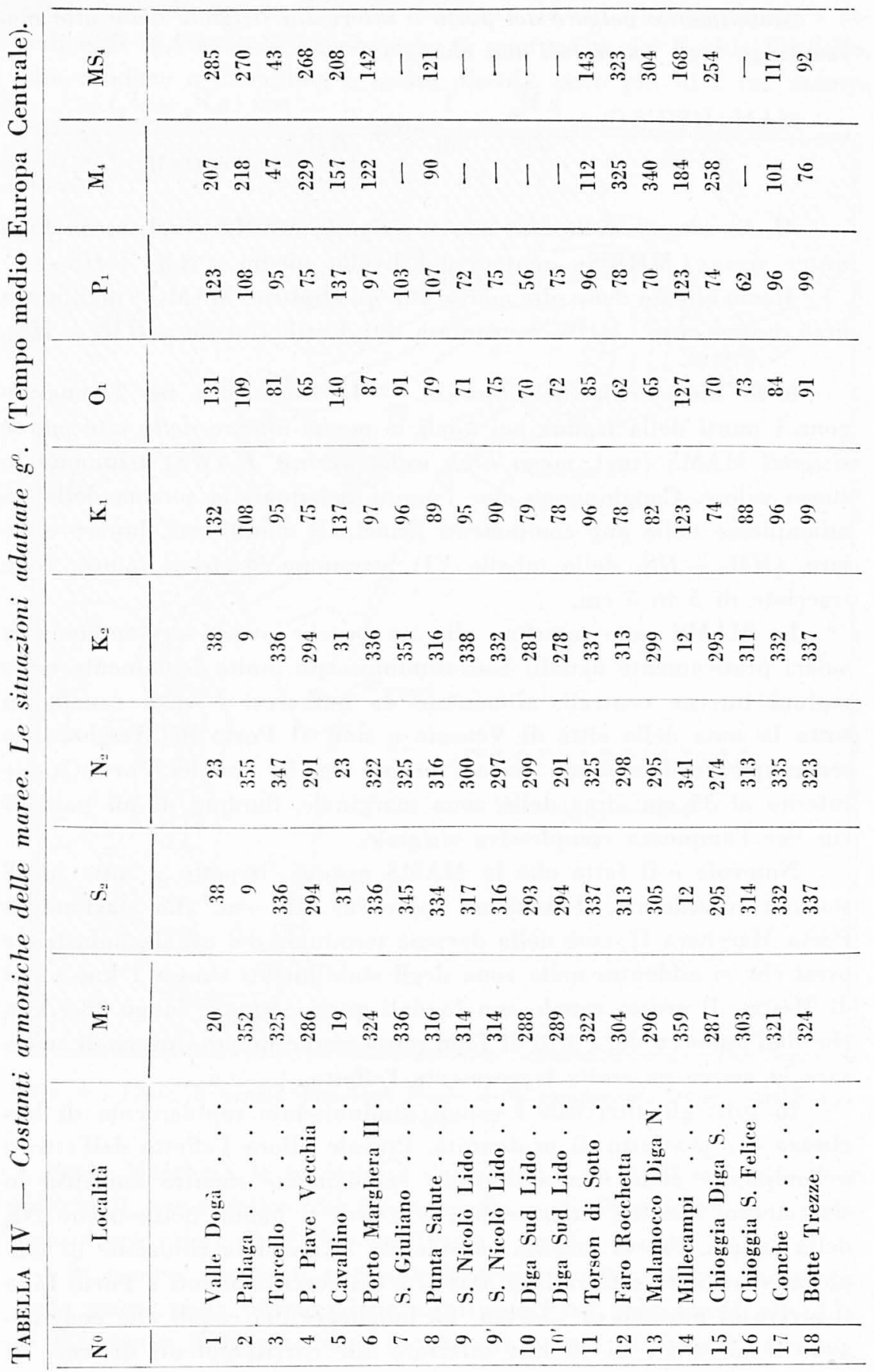


Stabilimento volgare del porto o intervallo sizigiale della alta marea (ingl. high water full and change):

$$
\operatorname{ISAM}(\mathrm{HWF} \& \mathrm{C})=\frac{x M_{2}}{29}-\frac{1}{30} \operatorname{arctg} \frac{\operatorname{sen}\left(x V_{2}-\cdots_{2}\right)}{\frac{H V_{2}}{H S_{2}}+\cos \left(\cdots M_{2}-\varkappa_{2}\right)}
$$

Media altezza delle alte maree sizigiali, MAMS (ingl. mean high water spring, MHWS), contata dal livello medio $=\boldsymbol{H} \boldsymbol{H} \boldsymbol{M}_{2}+\boldsymbol{H} \boldsymbol{S}_{2}$.

Media altezza cielle alte maree alle quadrature, MAMQ (ingl. mean high water neap, MHWN), contata dal livello medio $=H_{2} H_{2}-H S_{2}$.

6. Le linee di liguale ampiezza. -- Le linee della fig. 2 congriungono i punti della laguna nei quali le medie altezze dolle alte maree sizigiali MAMS (ingl. mean high water spring, MAWS) assumono lo stesso valore. Congriungono cioè $i$ punti nei quali la somma delle semiampiezze delle due componenti principali semidiurne, lunare e solare, $\left(H M_{2}+H S_{2}\right.$ della tabella $\left.\mathrm{Vl}\right)$, assumono lo stesso valore. Sono tracciate di 5 in $5 \mathrm{~cm}$.

Le MAMS sono massime alle tre bocche ove si presentano con valori praticamente uguali. Esse diminuiscono molto lentamente nelle regioni interne centrali, alimentate da numerosi e vasti canali. In tutta la zona della citta di Venezia e sino al Porto di Marghera, le semiampiezze decrescono verso l'interno dai $36 \mathrm{~cm}$ del Porto-Canale interno ai $35 \mathrm{~cm}$ rirca della zona marginale, dunque di un paio di em per l'ampiezza complessiva sizigiale.

Notevole è il fatto che la MAMS assume, rispetto a tutte le 18 stazioni considerate, il massimo valore di $38,7 \mathrm{~cm}$, alla stazione di Porto Marghera II, cioè nella darsena terminale del canale industriale ovest che si addentra nella zona degli stabilimenti sino a $1 \mathrm{~km}$ a sud di Mestre. Il grosso canale con fondali quasi costanti, lungo $18,5 \mathrm{~km}$, che dall'imbocco del Porto di Lido porta a questo sito, invece di smorzare la marea ne esalta leggermente l'effetto.

In tutti gli altri casi i canali diminuiscono rapidamente di larghezza e soprattutto di profondità. Prevale allora l'effetto dell'attrito e l'ampiezza della marea decresce rapidamente mentre aumenta lo sfasamento. Massimi smorzamenti e ritardi si hanno nell'estremo NE della laguna. Fra le località considerate la massima riduzione di ampiezza si registra a Cavallino. Dai $37,2 \mathrm{~cm}$ corrispondenti a Porto Lido si arriva al minimo di $15,9 \mathrm{~cm}$. La lunghezza dei canali che congriungono i due siti è di $16 \mathrm{~km}$, inferiore alle corrispondenti distanze di 
altre stazioni (per es. Lido-P. Marghera, la cui distanza lungo i canali è di $18,5 \mathrm{~km}$ ). Si ha però che la profondità del Canale Pordelio, che conduce a Cavallino, è molto piccola, poco più di $2 \mathrm{~m}$; mentre

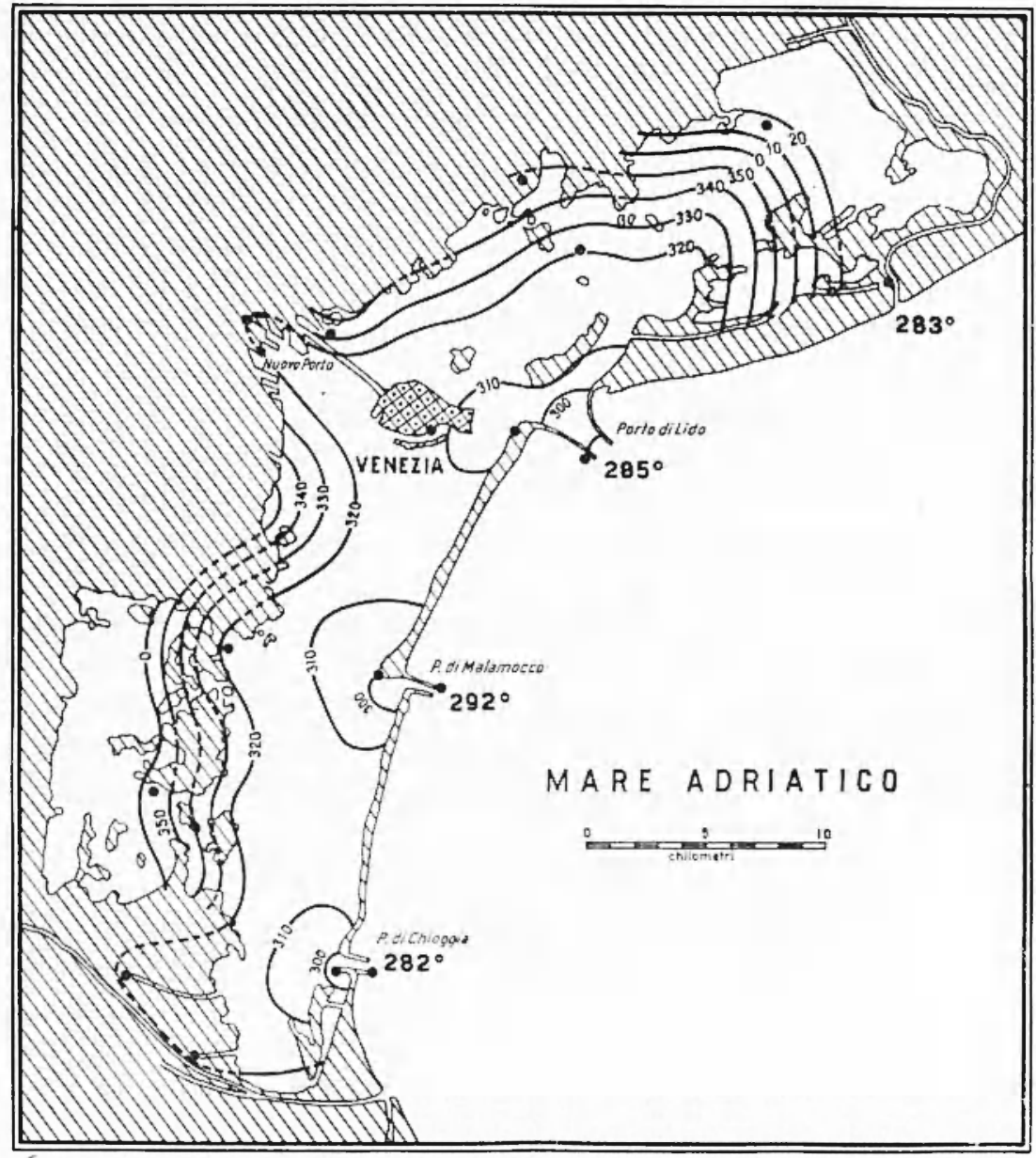

Fig. 4 - Linee di uguale situazione locale della componente lunare semidiurna principale $M_{2}$

a Porto Marghera le profondita dei canali sono di $9 \mathrm{~m}$. Nelle zone marginali, come nelle valli di Ingrassahò e di Dragajesolo si dovrebbero avere valori di MAMS sui $10 \mathrm{~cm}$ e anche minori.

Si osservi ancora il notevole decremento che subisce l'ampiezza della marea nella propagazione attraverso i tre porti canali di Lido, Malamocco e Chioggia. Sono lunghi, tra i due mareografi, km 3,5; 


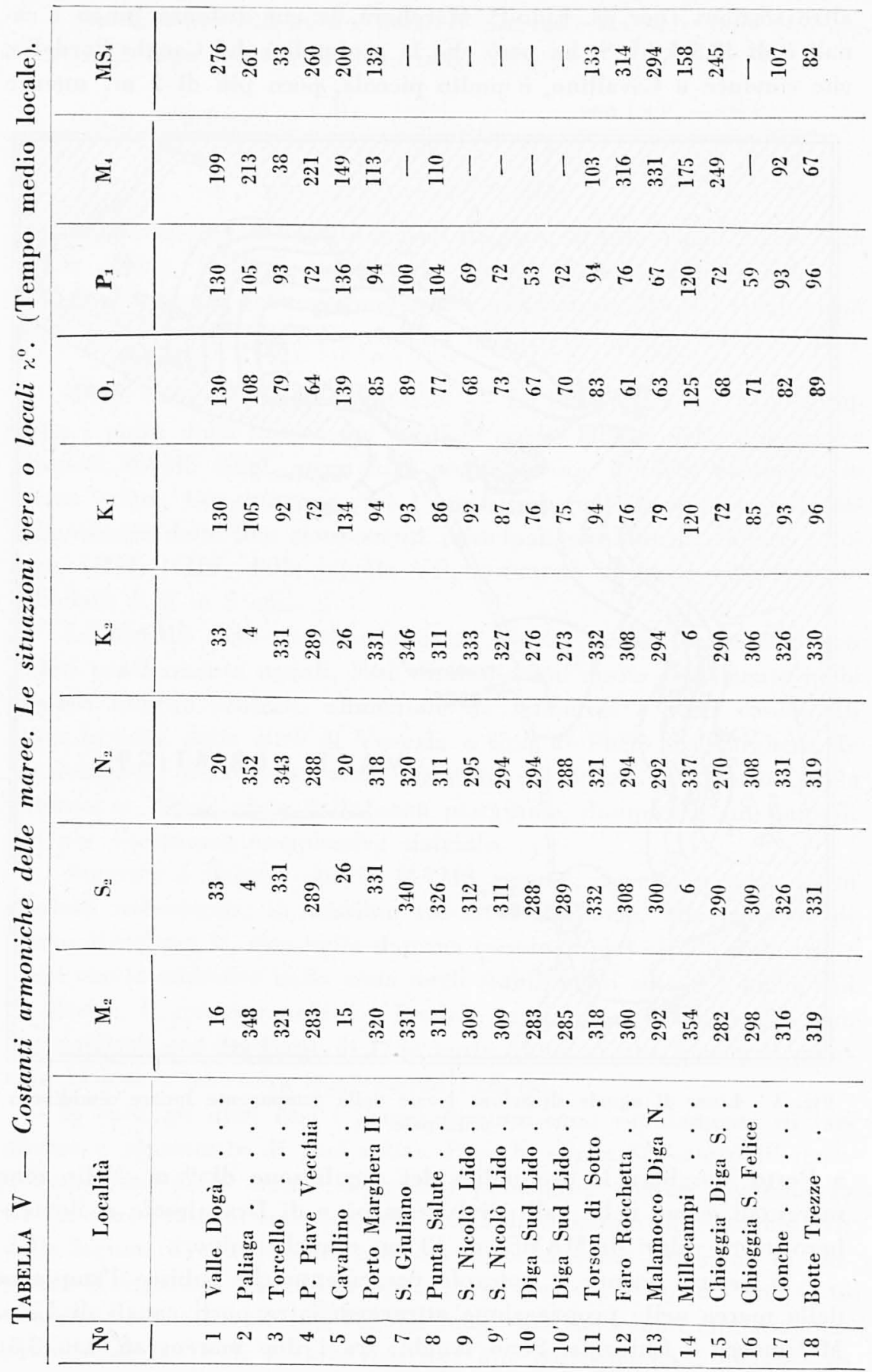


2,5 e 2,0. Le corrispondenti diminuzioni dei valori di MAMS risultano di 2,$6 ; 3,8$ e $2,6 \mathrm{~cm}$.

7. Le linee di uguale stabilimento. - Le linee della fig. 3 congiungono i punti nei quali lo stabilimento volgare del porto o intervallo sizigiale dell'alta marea ISAM (ingl. high water full and change, $\mathrm{HWF} \& \mathrm{C}$ ) assume lo stesso valore (tabella VI). Sono tracciate di 30 in 30 minuti. Gli stabilimenti minimi si banno alle tre bocche. Il passaggio attraverso queste produce ritardi che per i tre porti-canali di Lido, Malamoces e Chioggia risultano di 52, 19 e 32 minuti. Valori forti, lata la brevità del percorso, ma giustificati dalla grande massa acquea che deve passare per essi, alternativamente, nell'intervallo di poche ore.

Il valore dello stabilimento aumenta gradualmente dallinterno delle bocche verso i margini lagunari. Nella zona centrale assume valori crescenti fra gli stabilimenti 10,30 e 11.00. Venezia e le isole principali sono comprese tra queste due linee. Oltre la regione centrale, dove non arrivano più le grosse arterie, i valori dell'ISAM aumentano rapidamente, assumendo, nelle valli marginali, valori di $3-4$ ore maggiori di quelli che si hanno alle bocche. Nell'estremo NE, ciò nelle valli di Ingrassabò e Dragajesolo, il ritardo nella propagazione dovreble essere di 5 ore rispetto alle bocche e di 4 ore rispetto alla laguna centrale. Questi sono valori estrapolati dato che in quelle zone non vi sono stazioni mareografiche. Per le 18 località esaminate il massimo stabilimento corrisponde a Valle Dogà, con un ritardo rispetto a Venezia (Punta Salute) di $2^{\mathrm{l}} 37^{\mathrm{m}}$ e rispetto alla stazione di Lido Diga Sud di $3^{\mathrm{h}} 42^{\mathrm{m}}$.

8. Linee di uguale situazione locale. - Le linee della fig. $\&$ congiungono i punti nei quali la situazione vera o locale della componente lunare semidiurna principale $\mathrm{M}_{2}$ assume lo stesso valore. Sono state ottenute partendo dai valori della colonna di $\mathrm{M}$, della tabella V. Sono tracciate di $10^{\circ}$ in $10^{\circ}$. Hanno andamento simile alle linee di uguale stabilimento di porto segnate nella fig. 3 . Valgono pure per esse le stesse considerazioni. Curve analoghe si possono tracciare, con i dati della tabella. $V$, per le altre costituenti della marea.

Per la laguna assumono particolare interesse, specialmente da un punto di vista teorico, le linee corrispondenti alle componenti di acqua bassa: la sovramarea lunare quartodiurna $\mathrm{M}_{4}$ e l'onda composta $\mathrm{MS}_{4}$. I relativi valori, essendo molto piccoli, non sono stati sempre calcolati 
TABella VI

Costanti non armoniche delle stazioni della laguna di Venezia.

\begin{tabular}{|c|c|c|c|c|c|c|}
\hline $\mathbf{N}^{\circ}$ & Loc'alità & & & $\begin{array}{c}\text { ISAM } \\
\text { (MHWFSC) }\end{array}$ & $\begin{array}{l}\text { MM- HS: } \\
\text { (MHWS) }\end{array}$ & $\begin{array}{l}H_{2}-H S: \\
\text { (MHWN) }\end{array}$ \\
\hline 1 & Valle Doga & . . & & $0^{l} \cdot 43^{m}$ & $20,9 \mathrm{~cm}$ & $7,7 \mathrm{~cm}$ \\
\hline 2 & Paliaga . . . . . & . . & & 1210 & 29,6 & 10,0 \\
\hline$\overline{3}$ & Torcello : . . & . . & . & 1101 & 29,1 & 10,1 \\
\hline 4 & I'. l'iave Vecchial. & . . & . & $9+19$ & 35,8 & 8,0 \\
\hline 5 & Cavallino & . . & . & 0) 38 & 15,9 & $\mathbf{5 , 5}$ \\
\hline 6 & Porto Marghera II . & . . & . & 1109 & 38,7 & $10, \overline{3}$ \\
\hline 7 & S. Giuliano . . & . . & 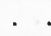 & 1131 & 36,9 & 10,1 \\
\hline 8 & Puntal salute. . & . . & . & $105 \cdot 4$ & 35,4 & 9,8 \\
\hline 9 & S. Nicolo Lido. & . . & . & $10+2$ & 34,0 & 8,8 \\
\hline $9^{\prime}$ & S. Nicoló Lido. . & . . & . & $10+1$ & 34,6 & 9,2 \\
\hline 10 & Dirgal Sud Lido. . & . . & . & 9.49 & $3 \pi, 2$ & 9,6 \\
\hline $100^{\circ}$ & Digal sud Lido. . & 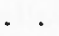 & . & 953 & 36,8 & 9,6 \\
\hline 11 & Torson di Sotto & 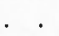 & . & 1109 & 36,0 & 5,6 \\
\hline 12 & Faro Rocchetta & . & . & 1029 & 33.7 & 8,3 \\
\hline 13 & Malamocco Diga N. & . . & . & 1010 & 37,5 & 9,5 \\
\hline 14 & Millecimpi . . & . . & . & 1215 & 26,4 & 7,4 \\
\hline 15 & Chioggia Digal $s$. & . & . & 953 & $3 \pi, 1$ & 9,2 \\
\hline 16 & Chioggia S. Felice & 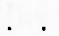 & . & 1025 & 34.8 & 10,4 \\
\hline 17 & Conche . . . . & . . & . & 1101 & 33,4 & 9,0 \\
\hline 18 & Botte Trezze . & . & 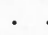 & 1113 & 33,0 & 8,6 \\
\hline
\end{tabular}

nelle prime analisi. Mancando cosi dati sufficienti, non si $\dot{e}$ ritenuto opportuno né di tracciarle né di discuterle. Per esequire un ricerca su questo particolare argomento bisognereble disporre di complete e omogenee analisi armoniche delle maree dei principali siti lagunari.

9. Considerazioni generali. - L'onda di marea si presenta quasi contemporaneamente alle tre bocche. Vi sono pochi minuti di ritardo nella propagazione da NNE a SSW. Le ampiezze possono considerarsi all'imbocco dei tre porti praticamente uguali, differendo tra di loro di pochi mm. Lungo i canali-porto si hanno notevoli diminuzioni di ampiezza e forti ritardi di propagazione. Nella laguna centrale, percorsa da numerosi e vasti canali, queste due ultime variazioni risultano molto meno ampie. Sono dell'ordine di pochi cm per le ampiezze e di $1 / 2$ ora per rli stabilimenti di porto. Nelle zone marcrinali, alimentate da canali poco profondi, rari, e che vanno morendo nelle valli, si ritorna nuovamente a rapidi decrementi di amplitudine e a forti ritardi. Ciò si osserva specialmente nella estremitì NE della laguna. 
Ogni restringimento, ma specialmente ogni diminuzione di profondita nei canali, smorza fortemente la propagazione della marea. Basta che un canale ahhia per pochi $\mathrm{km}$, talvolta anche meno di 5 , fondali di $2-3 \mathrm{~m}$, per ottenere sfasamenti di un'ora e diminuzioni di ampiezza del $30-40 \%$. Ben diversamente si propaga la marea in $\mathbf{~}$ canale di sezione quasi costante, o anche leggermente decrescente, ma che termina improvisamente con profondita e largheza poco diverse di quelle intermedic. L'onda incanalandosi si esalta e pur subendo in ritardo nella propayazione mantiene o aumenta l'ampiezza. Cio si osserva nella successione dei canali che conducono al Porto Industriale di Marghera. In questo sito, distante $18,5 \mathrm{~km}$ dall'imbocco del portocanale, le oscillazioni delle principali maree ragrgiungono aumenti dell'ordine del $5 \%$.

Nella citla di Venezia e nelle isole vicine, circondate e percorse da numerosi e profondi canali, tanto le ampiezze quanto i ritardi variano di poco fra loro, anche spostandosi verso l’interno lagunare: le prime diminuendo di pochi cm, i secondi aumentando di qualche decina di minuti. Le tabelle e le figure precisano quanto si è ora detto.

Questo lavoro è un primo sagrgio di ricerca sulla propagazione della marea nella laqruna di Venezia hasato esclusivamente sui valori delle costanti armoniche delle maree. La solidita dei dati di partenza, in senso di rigorositi, chiarezza e universalita, ì indubhia. Per uno studio più completo e particolareggiato occorrera disporre di costanti armoniche più omorrenee, più complete e relative ad un magrgior numero di località laqunari.

$$
\text { Trieste - Istituto Talassografico - Fobbraio 1952. (*) }
$$

\section{RIASSLVTO}

Si presentano le costanti armoniche e non armoniche delle marer. di 18 località della laguna di Venesia. Segue l'esame critico dei loro valori in relazione all'epoca dell'analisi e alla posizione della stazione. Sulla base di questi dati si tracciano, per la laguna di Venezia, le linee di uguale ampiezan (mean high water spring), di uguale stabilimento di porto (high water full and change) e, per la componente lunare

(*) Lavoro eseguito con il contributo del Consiglio Nazionale delle Ricerche p del Centro di Studi Talassografici del C.N.R. 
semidiurna principale $\mathrm{M}_{2}$, le linee di uguale situazione locale $\%$ Ai margini lagunari l'ampiezza della marea risulta ridotta del $50 \%$ e più, il ritardo di fase arriva sino a $3-4$ ore rispetto ai valori che si hanno all'imbocco dei porti-canali.

\section{$S U M M A R Y$}

In the article are expounded the harmonic and non-harmonic tidal constants for 18 different places of the Venitian Lagoon. This exposition is followed by critical remarks on their values in connection to the particular dates of the analysis and situation of the different stations. On the basis of these data, have been traced, for the Venitian Lagoon, the mean high water spring curves, the high water full \& change curves, and, for the principal lunar semi-diurnal component M.), the equal local situation $\%$. It results that, at the borders of the Lagoon, the mean high water spring curves, the high water full and the phase delay reaches the amount of 3 to 4 hours, in comparison to the values observed at the channel-harbour entrances.

\section{BIBLIOGRAFIA}

Comm. Maneocrafica It.: Verbali delle riunioni. Venezia, febbr. 1925.

Doodson A. T. - Warntng, H. D.: The Admiralty Tides Tables. Parl. III. Instruc. and Tables. Hydrogr. Departm. London, 1936.

Magnixi G. P.: Impianti mareografici eseguiti nella Laguna Veneta. Ricerche lagunari. Pubbl. N. 9. Istituto Veneto di Scienze, Lettere ed Arte, Venezia, 1908.

PoLli S.: Costanti armoniche e non armoniche della marea di Valle Doga, nella laguna di V'enezia. Atti Ist. Veneto di Sc. lett. arte, Cl. sc. mat. nat., 1942-13, T. CII, P. II, 579-583.

Polul S.: Il graduale aumento del livello del mare a Venezia, Trieste e Pola. Geofisica pura e appl., IX (1946), 1.2, 30-36.

Poll1 S.: Determinazione delle costanti armoniche e non armoniche delle maree per $i$ porti di Belvedere, Cortelazzo, Faro Rocchetta e Chioggia. Annali di Geofisica, II (1949), 3, 436.449 .

Pollı S.: Costanti armoniche e non armoniche delle maree di 4 localitù della la. guna di Venezia. (Paliağa, Torcello, Torson di Solto, Millecampi). Arch. di Oceanogr. e Limnol., VII 11950), 1, 17-27.

Tenani M.: Maree e correnti di marea. Ist. Idrogr. della Marina, 1935, 1940.

Vercelli F.: Il mare, $i$ laghi, $i$ ghiacciai. U.T.E.T., Torino, 1951.

ZiLle G. G.: Contributo sperimentale allo studio della propagazione della marea nella laguna di Venezia. Arch. di Oceanogr. e Limn. II, 2-3, 1942. 